\title{
Comparison of ultrasound-guided erector spinae plane block and thoracic paravertebral block for postoperative analgesia after video-assisted thoracic surgery: a prospective non-inferiority trial
}

\author{
Yasuko Taketa MD and Taro Fujitani MD
}

Department of Anesthesiology and Critical Care, Ehime Prefectural Central Hospital, Matsuyama, Japan

\section{Introduction}

Erector spinae plane block (ESPB) is a representative indirect thoracic paravertebral block (TPVB) method, reported by Forero et al. in 2016. ' In the clinical field, numerous studies have reported that ESPB could provide a favorable analgesic effect. $^{2}$ However, there have been few prospective trials comparing ESPB and TPVB. We hypothesized that ESPB might provide analgesia as effective as that of TPVB for thoracoscopic surgery. This study was designed to examine whether the analgesic effect of ESPB is non-inferior to that of TPVB for video-assisted thoracic surgery (VATS).

\section{Methods}

Eighty- eight patients scheduled for VATS were randomly allocated to the ESPB group or the TPVB group.

All the patients received continuous infusion of $0.2 \%$ levobupivacaine ( $8 \mathrm{~mL} /$ hour) after $20 \mathrm{~mL}$ of bolus injection.

- Primary outcome: numerical pain rating score (NRS) at rest 24 hours postoperatively

- Maximum acceptable difference (non-inferiority margin) was defined as 1.

- Secondary outcome: NRS on movement, amount of rescue fentanyl used, and the number of anesthetized dermatomes $6 \mathrm{~h}$ and $20 \mathrm{~h}$ after initiating infusion

\section{Results}

- We analyzed 40 patients in the TPVB group and 41 patients in the ESPB group.

- There were no differences in the baseline characteristics between the groups.

- NRS scores at rest were significantly higher in the ESPB group than in the TPVB group at rest at 1-, 2-, and 24-h postoperatively.

- $95 \%$ confidence interval of the differences in NRS scores at rest 24-h postoperatively could not demonstrate noninferiority of ESPB (Figure 1).

- No significant difference was obtained between the groups in NRS scores upon movement at any time point, the amount of rescue fentanyl use.

- The number of anesthetized dermatomes at parasternal region was significantly greater in the TPVB group than in the ESPB group $(p<0.0001)$ (Table 1$)$.

- All anesthesia procedures were conducted without complications or any adverse events.

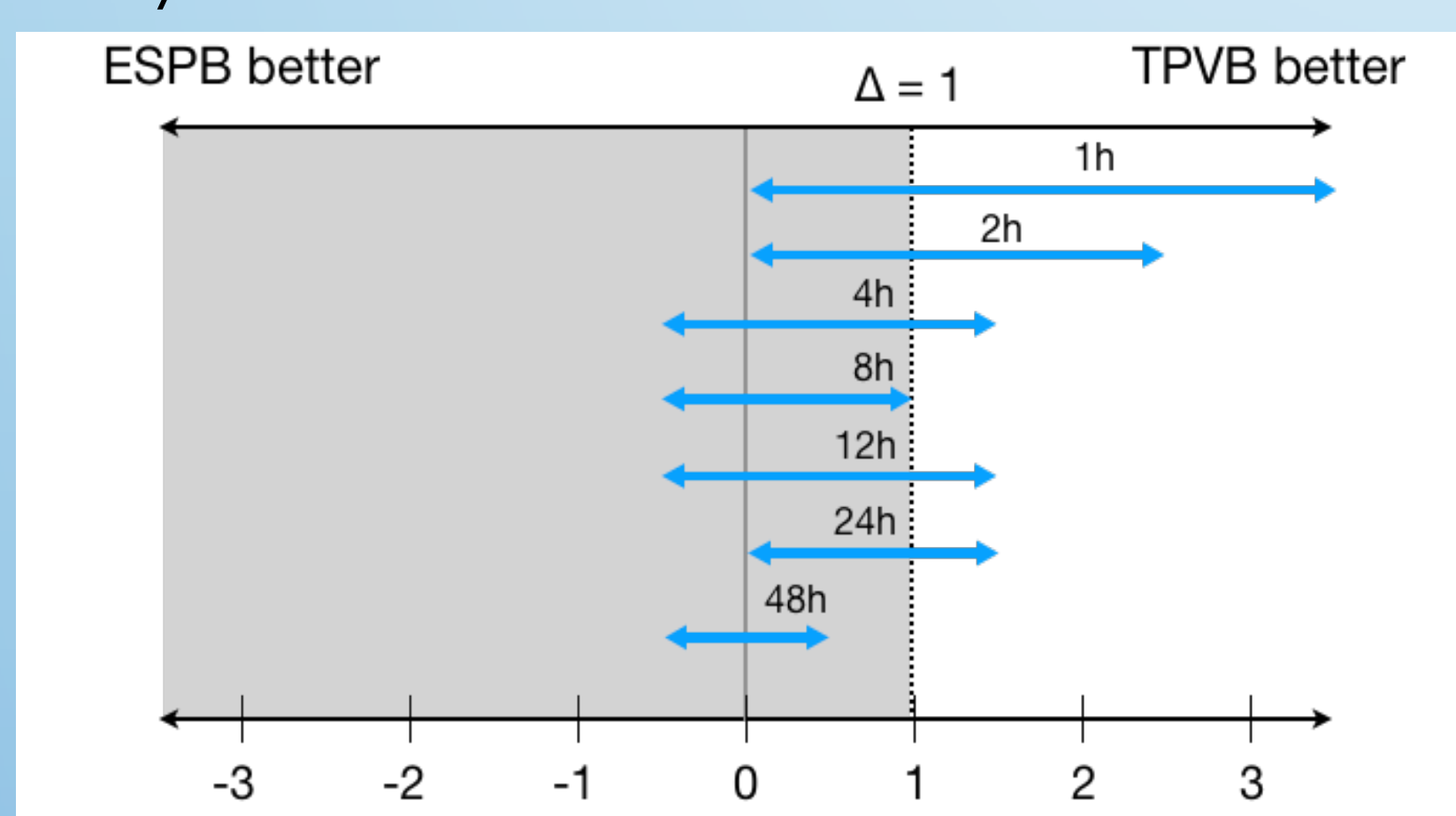

Figure 1 Median differences in NRS at rest (ESPB minus TPVB) Error bars representing $95 \%$ confidence intervals.

$\Delta$, margin of non-inferiority margin. Tinted area indicates zone of non-inferiority.

\begin{tabular}{|c|c|c|c|}
\hline & TPVB $(n=40)$ & ESPB $(n=41)$ & p value \\
\hline \multicolumn{4}{|c|}{ Paraspinal } \\
\hline $6 \mathrm{~h}$ & $3.8(1.5)$ & $4.1(1.5)$ & \multirow{2}{*}{0.87} \\
\hline $20 \mathrm{~h}$ & $4.1(1.4)$ & $3.8(1.5)$ & \\
\hline \multicolumn{4}{|c|}{ Anterior axillary } \\
\hline $6 \mathrm{~h}$ & $4.4(1.3)$ & $3.9(1.6)$ & \multirow{3}{*}{0.79} \\
\hline $20 \mathrm{~h}$ & $4.1(1.1)$ & $3.3(1.2)$ & \\
\hline \multicolumn{3}{|c|}{ Midclavicular } & \\
\hline $6 \mathrm{~h}$ & $4.2(1.1)$ & $3.3(1.2)$ & \multirow{3}{*}{0.06} \\
\hline $20 \mathrm{~h}$ & $4.2(1.2)$ & $3.4(1.1)$ & \\
\hline \multicolumn{3}{|c|}{ Parasternal } & \\
\hline $6 \mathrm{~h}$ & $3.1(1.3)$ & $0.3(0.4)$ & \multirow{2}{*}{$<0.0001$} \\
\hline $20 \mathrm{~h}$ & $3.2(1.4)$ & $0.6(0.6)$ & \\
\hline
\end{tabular}

Table 1 The number of anesthetized dermatomes after starting infusion Data are presented as mean (SD).

\section{Conclusions}

Our findings indicates that the anesthetized effect of ESPB for the anterior thoracoabdominal region could be weaker than that of paravertebral block and there was no clear evidence that ESPB achieved the equivalent analgesic effect to TPVB.

\section{References}

1. Forero $M$, et al. The erector spinae plane block: A novel analgesic technique in thoracic neuropathic pain. Reg Anesth Pain Med. 2016;41:621-627.

2. Tsui BCH, et al. The erector spinae plane (ESP) block: A pooled review of 242 cases. J Clin Anesth. 2019;53:29-34. 